\title{
NOTES ON THE THEORY OF THE SYNCHRONOUS MOTOR,
}

With Special Reference to the Phenomenon of Surging.

BY CHARLES PROTEUS STEINMETZ.

\section{I.}

While an induction motor at constant impressed voltage is fully determined as regards current, power factor, efficiency, etc., by one independent variable, the load or output, in the synchronous motor two independent variables exist, load and field excitation. That is, at constant impressed voltage the current, power factor, etc., of a synchronous motor may at the same power output be varied over a wide range by varying the field excitation, that is, the counter e.m.f. or "nominal induced e.m.f." Hence the synchronous motor may be utilized to fulfill two independent functions, to carry a certain load and to produce a certain wattless current, lagging by under-excitation, leading by over-excitation. Synchronous motors are, therefore, to a considerable extent used to control the phase relation and thereby the voltage, in addition to producing mechanical power.

The same applies to synchronous converters.

With given impressed e.m.f., field excitation or counter e.m.f. corresponding thereto, and load, determine by the usual theory all the quantities of the synchronous motor, as current, power factor, etc. Thus, if in diagram Fig. $1, \overline{O E}=e=$ e.m.f. consumed by the counter e.m.f. of the synchronous motor as corresponding to its field excitation, and if $P_{0}=$ output of motor (exclusive of friction and core loss and, if the exciter is driven by the motor, power consumed by the exciter), $i_{1}=P_{0} / e=$ 
energy component of current, represented by $\overline{O I_{1}}$, and the current vector therefore must terminate on a line $i$ perpendicular to $\overline{O I_{1}}$. If, then, $r=$ resistance and $x=$ reactance of the circuit between counter e.m.f. $e$ and impressed e.m.f. $e_{0}, \overrightarrow{O E}_{\mathrm{r}}=$ $i_{1} r=$ e.m.f. consumed by resistance, $\overrightarrow{O E}_{\mathrm{x}}=i_{1} x=$ e.m.f. consumed by reactance of the energy current $i_{1}$, hence $\overline{O E^{\prime}}{ }_{1}=$ e.m.f. consumed by impedance of the energy current $i_{1}$, and the impedance voltage of the total current lies on the perpendicular $e^{1}$ on $\overline{O E}_{1}{ }^{1}$. Producing $\overline{O E}_{1}=\overline{O E}$, and drawing an arc with the impressed e.m.f. $e_{0}$ as radius and $E_{1}$ as center, the point of intersection with $e^{1}$ gives the impedance voltage $\overline{O E}^{1}$, and corresponding thereto the current $\overline{O I}=i$; and completing the parallelogram $O E E_{0} E^{1}$ gives the impressed e.m.f. $\overline{O E}_{0}$.

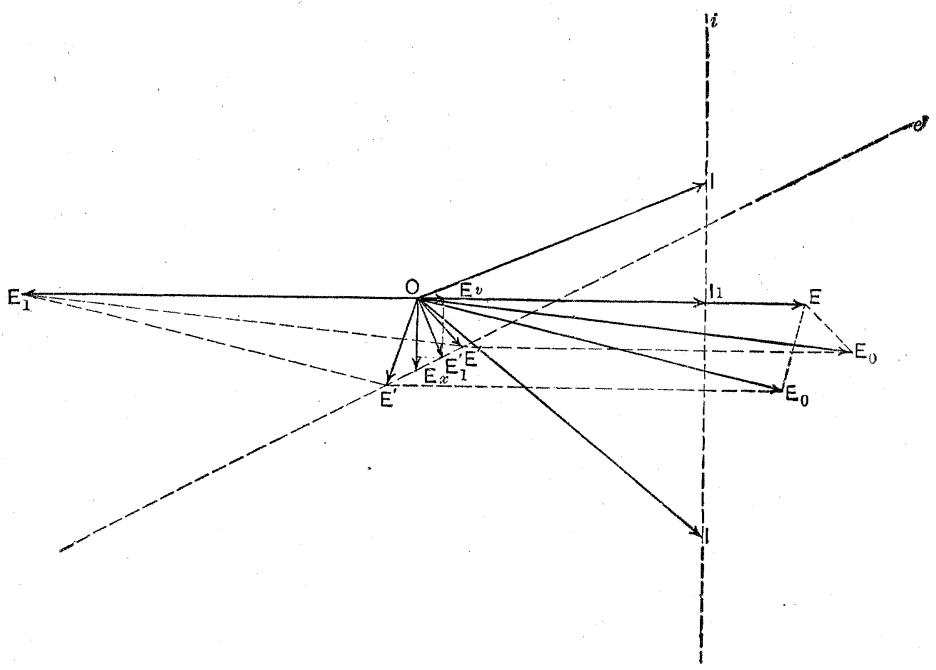

Fig. 1

Hence, by impressed e.m.f. $e_{0}$, counter e.m.f. $e$, and load $P_{0}$ the polar diagram is determined, and thereby the vectors $\overline{O I}=$ current, $\overline{O E_{0}}=$ impressed e.m.f., $\overline{O E}=$ counter e.m.f., ana their phase relation.

Or, in symbolic representation, let:

$E_{0}=e_{0}{ }^{1}+j e_{0}^{\prime \prime}=$ impressed e.m.f. $; e_{0}=\sqrt{e_{0}^{\prime 2}+e_{0}^{\prime \prime 2}}$

$E=e^{\prime}+j e^{\prime \prime}=$ e.m.f. consumed by counter e.m.f. $\sqrt{e^{\prime 2}+e^{\prime \prime 2}}$

$I=i=$ current, assumed as zero vector.

$Z=r-j x=$ impedance of circuit between $e_{0}$ and $e$. 
$Z$ is the synchronous impedance of the motor, if $e_{0}$ is its terminal voltage. It is the impedance of transmission line with transformers and motor, if $e_{0}$ is terminal voltage of generator, and $Z$ is synchronous impedance of motor and generator, plus impedance of line and transformer, if $e_{0}$ is the nominal induced e.m.f. of the generator (corresponding to its field excitation).

It is, then:

$$
E_{0}=E+i Z
$$

or:

$$
e_{0}^{\prime}+i e_{0}^{\prime \prime}=e^{\prime}+j e^{\prime \prime}+i r-j i x
$$

and, resolved:

$$
\left\{\begin{array}{l}
e_{0}^{\prime}=e^{\prime}+i r \\
e_{0}^{\prime \prime}=e^{\prime \prime}-i x
\end{array}\right.
$$

The power output of the motor (inclusive of friction and core loss, and, if the exciter is driven by the motor, power consumed by exciter) is current times energy component of induced e.m.f., or:

$$
P_{0}=e^{\prime} i
$$

Hence, the calculation of the motor, from power output $P_{0}$, occurs by the equation:

Chosen: $i=$ current.

(7) $e^{\prime}=P_{0} / i$

(5) $e_{0}^{\prime}=e^{\prime}+i r$

(1) $e_{0}^{\prime \prime}= \pm \sqrt{e_{0}^{2}-e_{0}{ }^{2}}$

(6) $e^{\prime \prime}=e_{0}^{\prime \prime}+i x$

(2) $e=\sqrt{e^{\prime 2}+e^{\prime 2}}$

That is, at given power $P_{0}$, to every value of current $i$ correspond two values of the counter e.m.f. $e$ (and hence the field excitation).

For illustration are plotted in Fig. 2, curves giving the current $i$ as function of the counter e.m.f. $e$, at constant power $P_{0}$, the so-called "synchronous motor phase characteristics," for the values:

$$
\begin{aligned}
e_{0} & =2,200 \text { volts } \\
Z & =1-4 j \mathrm{ohms}
\end{aligned}
$$

and; $\quad P_{0}=20,200,400,600,800,1,000 \mathrm{k} . w$. output. 
The five equations of the synchronous motor:

$$
\begin{aligned}
& \text { (1) } e_{0}^{2}=e_{0}{ }^{2}+e_{0}{ }^{\prime \prime 2} \\
& \text { (2) } e^{2}=e^{\prime 2}+e^{\prime 2} \\
& \text { (7) } P_{0}=e^{\prime} i \\
& \text { (5) } e_{0}^{\prime}=e^{\prime}+i r \\
& \text { (6) } e_{0}^{\prime \prime}=e^{\prime \prime}-i x
\end{aligned}
$$

determine the five quantities: $e_{0}{ }^{\prime}, e_{0}{ }^{\prime \prime}, e^{\prime}, e^{\prime \prime}, e$, as functions of $P_{0}$ and $i$.

The condition of zero phase displacement, or unity power factor at the impressed e.m.f. $e_{0}$, is:



FIG. 2.

$$
e_{0}^{\prime \prime}=0
$$

hence:

$$
e_{0}^{\prime}=e_{0} \text {, and }
$$

(6) $e^{\prime \prime}=i x$

(5) $e^{\prime}=e_{0}-i r$

hence:

$$
e^{2}=\left(e_{0}-i r\right)^{2}+i^{2} x^{2}
$$

a quadratic equation, the hyperbola of unity power factor, shown as dotted line in Fig. 2. 
In this case, the power is found by substituting $e^{\prime}=e_{0}-i r$ in $P_{0}=e^{\prime} i$, as:

$$
P_{0}=e_{0} i-i^{2} r
$$

or:

$$
i=\frac{e_{0}}{2 r}\left\{1 \pm \sqrt{1-\frac{4 r P_{0}}{e_{0}{ }^{2}}}\right\}
$$

As maximum output of the synchronous motor follows herefrom:

$$
\sqrt{1-\frac{4 r P_{0}}{e_{0}^{2}}=0}
$$

in above instance:

$$
P_{0}=1,210 \mathrm{k} . \mathrm{w} . \text { at: } i=1,100 \text { amps. }
$$

The curve of unity power factor (8) divides the synchronous motor phase characteristics into two sections, one, for lower $e$, with lagging, the other with leading current.

The study of these phase characteristics, Fig. 2, gives the best insight into the behavior of the synchronous motor under conditions of steady operation.

Of special interest are the load curves of the synchronous motor, or curves giving, at constant excitation: $e=$ constant, the current, power factor, efficiency and apparent efficiency as function of the load or output $P=P_{0}-$ (friction + core loss + excitation). Such load curves are represented in Figs. 3, 4, 5, 6, 7 for $e=1,600,2,800,2,000,2,400,2,180$ volts. They are derived from Fig. 2 as the intersection of the curves $P_{0}=$ constant with the vertical lines $e=$ constant.

Hence, while an induction motor has one load curve only, a synchronous motor has an infinite series of load curves, depending upon the value of $e$.

As seen, for low values of $e(e=1,600$, under excitation, Fig. 3 ), the load curves are similar to those of an induction motor. The current is lagging, the power factor rises from a low initial value to a maximum, and then falls again. With increasing excitation ( $e=2,000$, Fig. 5$)$ the power factor curve rises to values beyond those available in induction motors, approaches and ultimately touches unity, and with still higher excitation $(e=2,180$, Fig. 7) two points of unity power factor exist, at $P=20$ and $P=450 \mathrm{k}$.w. output, which are separated by a range with leading current, while at very low and very high load 
FIG. 3.

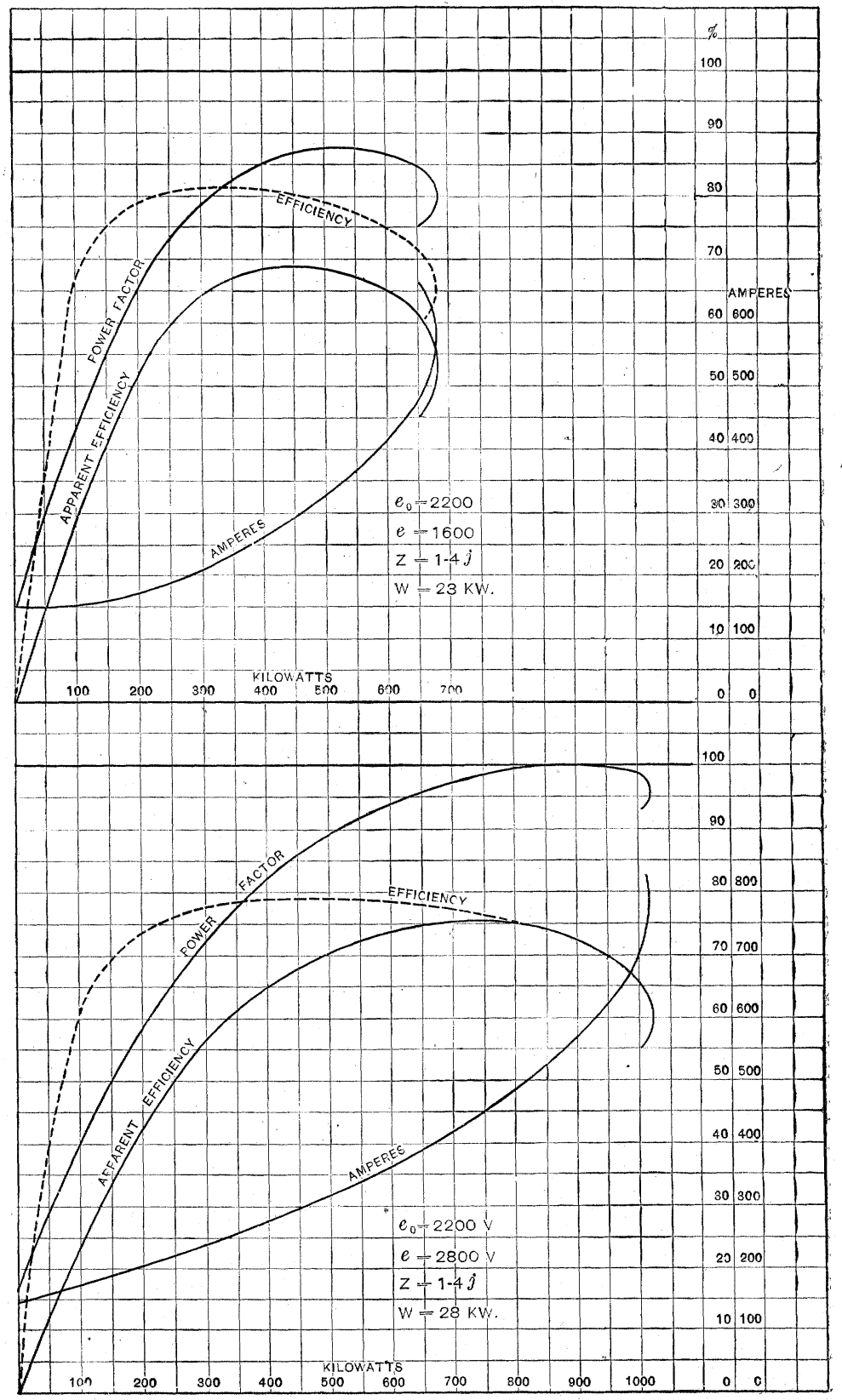

FIG. 4. 
the current is lagging. The first point of unity power factor moves toward $P=O$, and then disappears, that is, the current becomes leading already at no load, and the second point of unity power factor moves with increasing excitation toward higher loads, from $P=450 \mathrm{k}$.w at $e=2,180$ in Fig. 7, to $P=$ $700 \mathrm{k} . \mathrm{w}$. at $e=2,400$ and $P=900 \mathrm{k} . \mathrm{w}$. at $e=2,800$, while the power factor and thereby the apparent efficiency decrease at light loads. The maximum output increases with the increase of excitation and almost proportionally thereto.

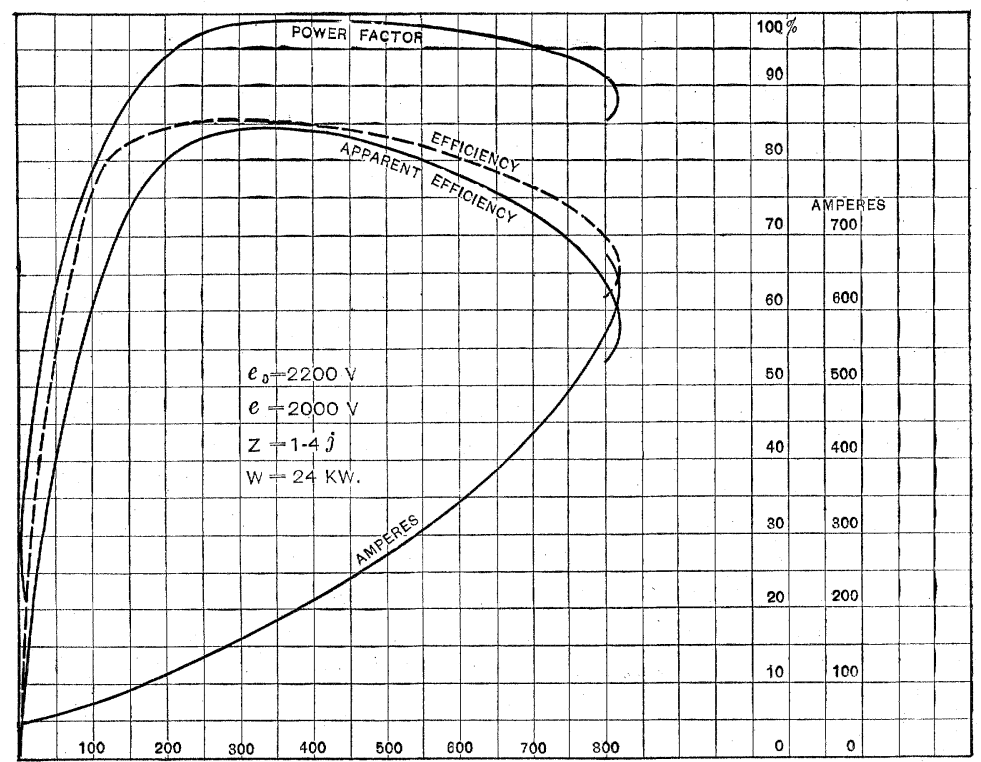

FIG. 5 .

It is interesting that at $e=2,180$, the power factor is practically unity over the whole range of load up to near the maximum output. It is shown once more in Fig. 7 with increased scale of the ordinates. A synchronous motor at constant excitation can, therefore, give practically unity power factor for all loads.

The resistance $r=1 \mathrm{ohm}$ is assumed so as to represent a synchronous motor inclusive of transmission line, with about $9 \%$ loss of energy in the line at $400 \mathrm{k}$.w. output.

The friction and core losses are assumed as $20 \mathrm{k}$. w., the excitation as $4 \mathrm{k}$.w. at $e=2,000$.

Considering the intersections of a horizontal line with the 
constant power curves of Fig. 2, gives the characteristic curves of the synchronous motor when operating on constant current. Such curves are shown for $i=300$ in Fig. 8. They illustrate that at the same impressed voltage with the same current input, the power output of the synchronous motor may vary over a wide range, and also that for each value of power output two points exist, one with lagging, the other with leading current.

As regards phase characteristics and load characteristics, the same applies to the synchronous converter as to the synchronous motor, except that in the former the continuous cur-

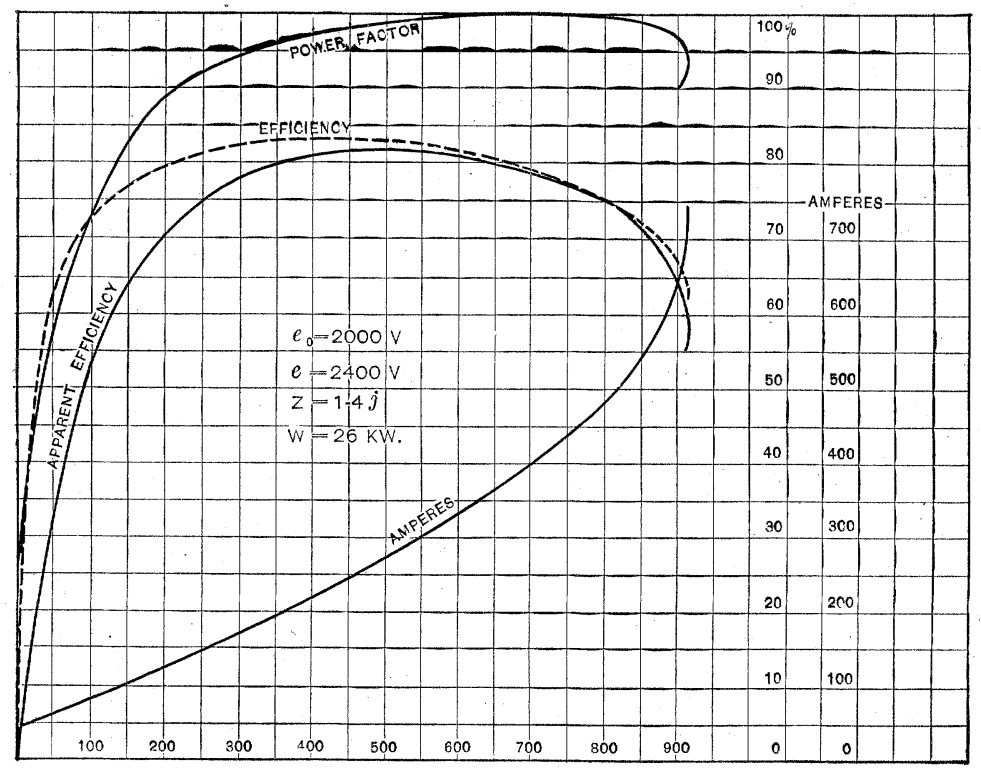

FIG. 6.

rent output affords a means of automatically varying the excitation with the load.

\section{II.}

In the preceding theory of the synchronous motor the assumption has been made that the mechanical output of the motor equals the power developed by it. This is the case only if the motor runs at constant speed. If, however, it accelerates, the power input is greater, if it decelerates, less than the power output, by the power stored in and returned by the momentum. Obviously, the motor can neither constantly accelerate nor 
decelerate, without breaking out of synchronism, since the counter e.m.f. $e$ inust remain within a quarter wave of the impressed e.m.f. $e_{0}$, assuming the latter as of constant frequency.

If, for instance, at a certain moment the power produced by the motor exceeds the mechanical load (as, for instance, in the moment of throwing off a part of the load), the excess power is

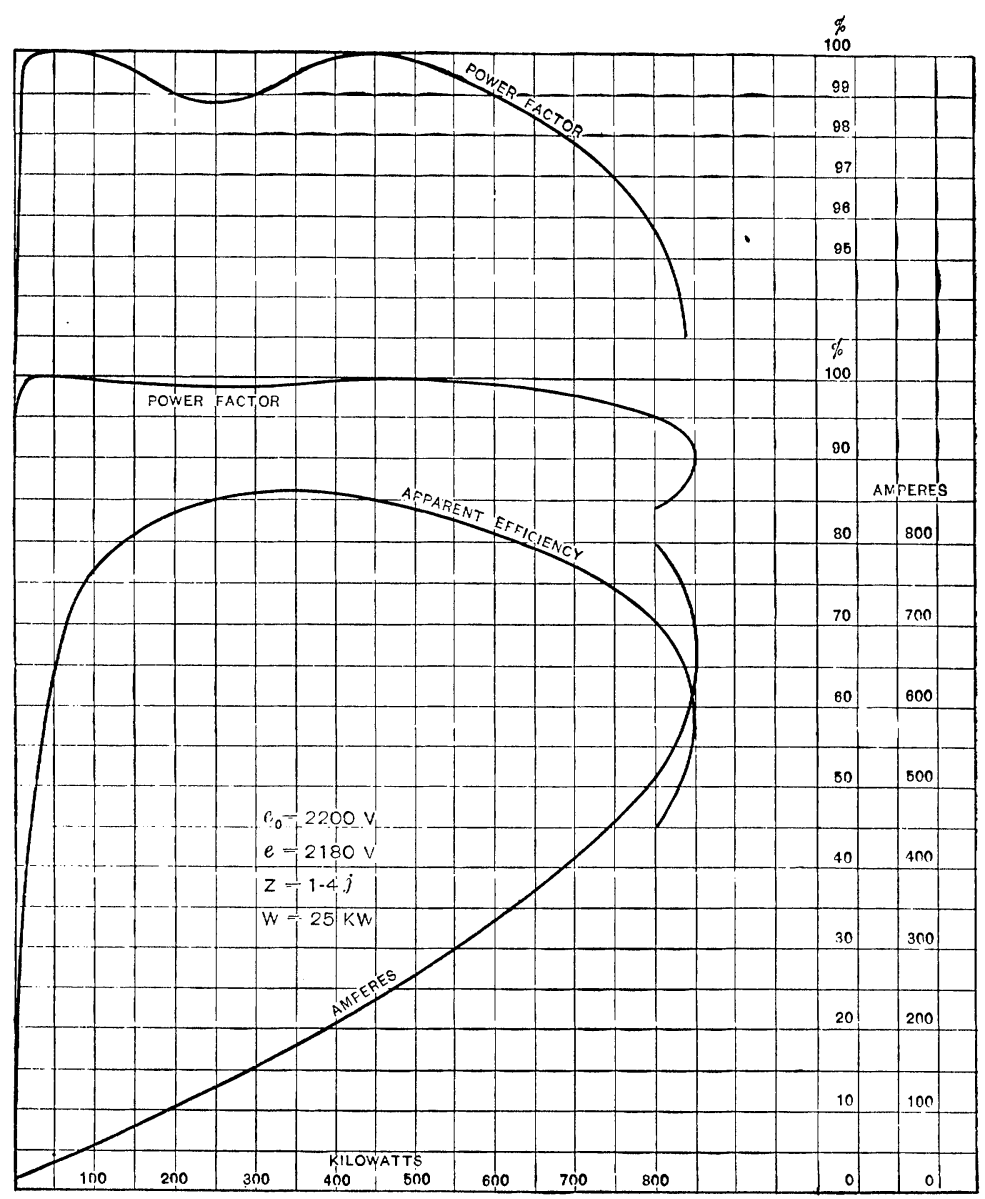

FIG. 7.

consumed by the momentum as acceleration, causing an increase of speed. The result thereof is that the phase of the counter e.m.f. $e$ is not constant, but the vector $e$ (in Fig. 1) moves backward to earlier time, or counter-clockwise, at a rate ciepending upon the momentum Thereby the current changes 
and the power developed changes and decreases. As soon as the power produced equals the load, the acceleration ceases, but the vector $e$ still being in motion, due to the increased speed, further reduces the power, causing a retardation and thereby a decrease of speed, at a rate depending upon the mechanical

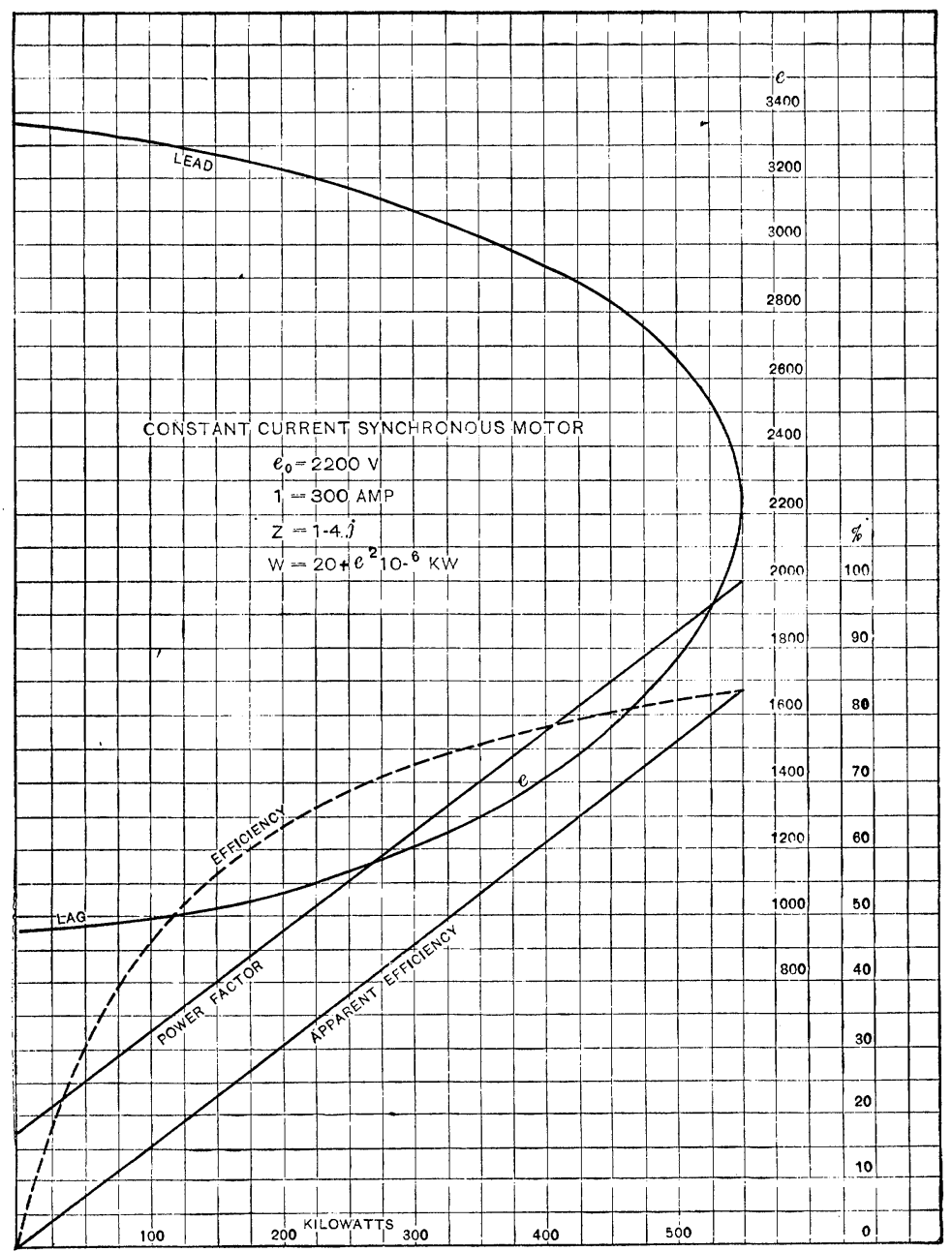

FIG. 8.

momentum. In this manner a periodic variation of the phase relation between $e$ and $e_{0}$, and corresponding variation of speed and current occurs, of an amplitude and period depending upon the circuit conditions and the mechanical momentum. 
If the amplitude of this pulsation has a positive decrement, that is, is decreasing, the motor assumes after a while a constant position of $e$ regarding $e_{0}$, that is to say, its speed becomes uniform. If, however, the decrement of the pulsation is negative, an infinitely small pulsation will continuously increase in amplitude, until the motor is thrown out of step,or the decrement becomes zero, by the power consumed by forces opposing the pulsation, as anti-surging devices. If the decrement is zero, a pulsation started once will continue indefinitely at constant amplitude. This phenomenon, a surging by what may be called electromechanical resonance, must be taken into consideration in a complete theory of the synchronous motor.

Let:

$E_{0}=c_{0}=$ impressed e.m.f., assumed as zero vector.

$E=e(\cos \beta+j \sin \beta)=$ e.m.f. consumed by counter e.m.f. of motor where $\quad \beta=$ phase angle between $E_{0}$ and $E$.

Let:

$Z=r-j x, z=\sqrt{r^{2}}+x^{2}=$ impedance of circuit between $E_{0}$ and $E$, and: $\tan \alpha=x / r$

The current in the system is:

$$
\begin{gathered}
I_{0}=\frac{e_{0}-E}{z}=\frac{e_{0}-e \cos \beta-j e \sin \beta}{r-j x} \\
=(1 / z)\left\{\left[e_{0} \cos \alpha-e \cos (\alpha+\beta)\right]+j\left[e_{0} \sin \alpha-e \sin (\alpha+\beta)\right]\right\}
\end{gathered}
$$

The power developed by the synchronous motor is:

$$
\begin{aligned}
P_{0}= & {[E I]^{1} } \\
= & (e / z):\left[\cos \beta\left[e_{0} \cos \alpha-e \cos (\alpha+\beta)\right]+\sin \beta\left[e_{0} \sin \alpha-e\right.\right. \\
& \sin (\alpha+\beta)]_{i}^{\prime} \\
= & (e / z)\left\{\left[e_{0} \cos (\alpha-\beta)-e \cos \alpha\right]\right\}
\end{aligned}
$$

If, now, a pulsaton of the synchronous motor occurs, resulting in a change of the phase relation $\beta$ between the counter e.m.f. $e$ and the impressed e.m.f. $e_{0}$ (the latter being of constant frequency, thus constant phase), by an angle $\delta$, where $\delta$ is a periodic function of time, of a frequency very low compared with the impressed frequency, then the phase angle of the counter e.m.f. $a$ is: $\beta+\delta$; and the counter e.m.f. is: 


$$
E=e\{\cos (\beta+\delta)+j \sin (\beta+\delta)\}
$$

hence the current:

$$
\begin{aligned}
& I=(1 / z)\left\{\left[e_{0} \cos \alpha-e \cos (\alpha+\beta+\delta)\right]+j\left[e_{0} \sin \alpha-e \sin \right.\right. \\
& \quad(\alpha+\beta+\delta)]\} \\
& =I_{0}+\frac{2 e}{z} \sin \frac{\delta}{2}\left\{\sin \left(\alpha+\beta+\frac{\delta}{2}\right)-j \cos \left(d+\beta+\frac{\delta}{2}\right)\right\}
\end{aligned}
$$

the power:

$$
\begin{aligned}
& P=e / z\left\{e_{0} \cos (\alpha-\beta-\delta)-e \cos \alpha\right\} \\
& =P_{0}+\frac{2 e e_{0}}{z} \sin \frac{\delta}{2} \sin \left(a-\beta-\frac{\delta}{2}\right)
\end{aligned}
$$

Let now:

$v_{0}=$ mean velocity (linear, at radius of gyration) of synchronous machine

$s=$ slip, or decrease of velocity, as fraction of $v_{0}$, where $s$ is a (periodic) function of time; hence:

$v=v_{0}(1-s)=$ actual velocity, at time $t$.

During the time element $d t$, the position of the synchronous motor armature regarding the impressed e.m.f. $e_{0}$, and thereby the phase angle $\beta+\delta$ of $e$, changes by:

$$
\begin{aligned}
d \delta & =2 \pi N s d t \\
& =s d \varphi
\end{aligned}
$$

where:

$$
\varphi=2 \pi N t
$$

and:

$$
N=\text { frequency of impressed e.m.f. } e_{0}
$$

Let:

$m$ = mass of revolving machine elements, and

$M_{0}=\frac{1}{2} m v_{0}^{2}=$ mean mechanical momentum, reduced to joules or watt-seconds, then the momentum at time $t$ and velocity $v=v_{0}(1-s)$ is :

$$
M=\frac{1}{2} m v_{0}^{2}(1-s)^{2}
$$

and the change of momentum during the time element $d t$ is:

$$
\frac{d M}{d t}=-m v_{0}^{2}(1-s) \frac{d s}{d t}
$$

hence, for small values of $s$ : 


$$
\begin{aligned}
\frac{d M}{d t} & =-m v_{0}{ }^{2} \frac{d s}{d \varphi} \frac{d \varphi}{d t} \\
& =-2 M_{0} \frac{d s}{d \varphi} \frac{d \varphi}{d t}
\end{aligned}
$$

since:

it is:

$$
\begin{gathered}
\frac{d \varphi}{d t}=2 \pi N, \text { and frcm (15): } \\
s=\frac{d \delta}{d \varphi} \\
\frac{d s}{d \varphi}=\frac{d^{2} \delta}{d \varphi^{2}}
\end{gathered}
$$

$$
\frac{d M}{d t}=-4 \pi N M_{0} \frac{d^{2} \delta}{d \varphi^{2}}
$$

Since, as discussed, the change of momentum equals the difference between produced and consumed power, the excess of power being converted into momentum, it is:

$$
P-P_{0}=\frac{d M}{d t}
$$

and, substituting (14) and (17):

$\frac{\epsilon e_{0}}{z} \sin \frac{\delta}{2} \sin \left(\alpha-\beta-\frac{\delta}{2}\right)+2 \pi N M_{0} \frac{d^{2} \delta}{d \varphi^{2}}=0$

Assuming $\delta$ as a small angle, that is, considering only small oscillations, it is:

$$
\begin{aligned}
& \sin \frac{\delta}{2}=\frac{\delta}{2} \\
& \sin \left[\alpha-\beta-\frac{2}{\delta}\right]=\sin (\alpha-\beta)
\end{aligned}
$$

hence, substituted in (18):

$$
\frac{e e_{0}}{z} \delta \sin (a-\beta)+4 \pi N M_{0} \frac{d^{2} \delta}{d \varphi^{2}}=0
$$

and, substituting:

it is:

$$
a=\frac{e e_{0} \sin (a-\beta)}{4 \pi N z M_{0}}
$$

$$
a \delta+\frac{d^{2} \delta}{d \varphi^{2}}=0
$$

This differential equation is integrated by:

$$
\delta=A \varepsilon C_{\phi}
$$

which, substituted in (22) gives:

$$
a A \varepsilon^{C} \dot{ }+A C^{2} C_{\varphi}=0
$$




$$
\begin{gathered}
a+C^{2}=0 \\
C= \pm \sqrt{-a}
\end{gathered}
$$

(1) If $a<0$, it is:

$$
\dot{\delta}=A_{1} \varepsilon+m \phi+A_{2} \varepsilon-m \varphi
$$

where

$$
m=\sqrt{-a}=\sqrt{\frac{e e_{0} \sin (\beta-a)}{4 \pi N z M_{0}}}
$$

Since in this case, $\varepsilon+m \phi$ is continually increasing: the synchronous motor is unstable. That is, without oscillation it drops out of step, if $\beta>a$

(2) If $a>0$, it is, denoting:

$$
\begin{gathered}
n=+\sqrt{ } a=+\sqrt{\frac{e e_{0} \sin (a-\beta)}{4 \pi N z M_{0}}} \\
\delta=A_{1} \varepsilon+j n \phi+A_{2} \varepsilon^{-j n \phi}
\end{gathered}
$$

or, substituting for $\varepsilon^{+j n \phi}$ and $\varepsilon^{+j n \phi}$ the trigonometric functions:

$$
\delta=\left(A_{1}+A_{2}\right) \cos n \varphi+j\left(A_{1}-A_{2}\right) \sin n \varphi
$$

or:

$$
\delta=B \cos (n \varphi+\gamma)
$$

That is, the synchronous motor is in stable equilibrium, when oscillating with a constant amplitude $B$, depending upon the initial conditions of oscillation, and a period, which for small oscillations gives the frequency of oscillation:

$$
\begin{gathered}
N_{0}=n N \\
=\sqrt{\frac{N e e_{0} \sin (\alpha-\beta)}{4 \pi z \bar{M}_{0}}}
\end{gathered}
$$

In the instance given in Part $I$ it is:

$e_{0}=2,200$ volts. $Z=1-4 j$ ohms, or: $z=4.12 ; a=76^{\circ}$

Let the machine, a 16 -polar 60 -cycle $400 \mathrm{k}$.w. revolving field synchronous motor, have the radius of gyration of $20^{\prime \prime}$, a weight of the revolving part of $6,000 \mathrm{lbs}$.

The momentum then is: $M_{0}=850.000$ joules. 
Deriving the angles $\beta$, corresponding to given values of output $P$ and excitation $e$, from the polar diagram, or from the symbolic representation, and substituting in (26), gives the frequency of oscillation:

$P=0$ :

$e=1600$ volts $\beta=-2^{0} \quad N_{0}=2.17$ cycles, or 130 periods per min.

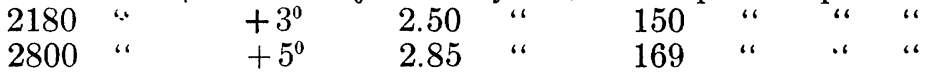

$P=400 \mathrm{k} . \mathrm{w}$.

$e=1600$ volts $\beta=33^{\circ} \quad N_{0}=1.90$ cycles, or 114 periods per min.

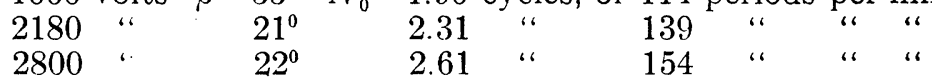

As seen, the frequency of oscillation does not vary much with the load and with the excitation. It slightly decreases with increase of load, and it increases with increase of excitation.

In this instance, only the momentum of the motor has been considered, as would be the case for instance in a synchronous converter.

In a direct connected motor-generator set, assuming the momentum of the direct current generator armature equal to $60 \%$ of the momentum of the synchronous motor, the total momentum is: $M_{0}=1,360,000$ joules, hence, at no load:

$$
P=0
$$

$e=1,600$ volts: $N_{0}=1.72$ cycles, or 103 periods per min.

$\begin{array}{lllll}1.98 & \text { ، } & 119 & \text { ، } & \text { “ } \\ 1.23 & \text { “ } & 134 & \text { ، } & \end{array}$

III.

In the preceding discussion of the surging of synchronous machines, the assumption has been made, that the mechanical power consumed by the load is constant, and that no damping or anti-surging devices were used.

The mechanical power consumed by the load varies, however, more or less with the speed, approximately proportional to the speed if the motor directly drives mechanical apparatus, as pumps, etc., and at a higher power of the speed if driving direct current generators, or as synchronous converter, especially when in parallel, with other direct current generators. Assuming, then, in the general case the mechanical power consumed by the load to vary, within the narrow range of speed variation considered during the oscillation, as the $p$ th power of the speed, in 
the preceding equation instead of $P_{0}$ is to be substituted: $P_{0}$ $(1-s)^{p}=P_{0}(1-p s)$.

If anti-surging devices are used, and even without these in machines in which eddy currents can be induced by the oscillation of slip, in solid field poles, etc., a torquc is produced more or less proportional to the deviation of speed from synchronism. This power assumes the form $P_{1}=c^{2} s$, where $c^{2}$ is a function of the conductivity of the eddy current circuit and the intensity of the magnetic field of the machine. $c^{2}$ is the power which would be required to drive the magnetic field of the motor through the circuits of the anti-surging device at full frequency, if the same relative proportions could be retained at full frequency as at the frequency of $\operatorname{slip} s$. That is, $P_{1}$ is the power produced by the motor as induction machine at slip $s$. Instead of $P$, the power generated by the motor, has to be substituted then in the preceding equations the value $P+P_{1}$.

The equation (18) then assumes the form:

$$
P+P_{1}-P_{0}(1-p s)=\frac{d M}{d t}
$$

or:

$$
\left(P-P_{0}\right)+\left(P_{1}+p P_{0} s\right)=\frac{d M}{d t}
$$

or, substituting (17) and (14)

$$
\begin{gathered}
2 e e_{0} \sin \frac{\delta}{2} \sin \left[\alpha-\beta \frac{\delta}{2}\right]+\left(c^{2}+p P_{0}\right) \frac{d \delta}{d \varphi}+ \\
4 \pi N M_{0} \frac{d^{2} \delta}{d \varphi^{2}}=0
\end{gathered}
$$

and, for small values of $\delta$ :

$$
\begin{gathered}
a \delta+2 b \frac{d \delta}{d \varphi}+\frac{d^{2} \delta}{d \varphi^{2}}=0 \\
a=\frac{e e_{0} \sin a-\beta}{4 \pi N z M_{0}} \\
b=\frac{c^{2}+p P_{0}}{8 \pi N M_{0}}
\end{gathered}
$$

Of these two terms $b$ represents the consumption, $a$ the oscillation of energy by the pulsation of phase angle $\beta$. $b$ and $a$ thus have a similar relation as resistance and reactance in alternating current circuits, or in the discharge of condensers. $a$ is the säme term as in part II. 
Differential equation (29) is integrated by: which, substituted in (29) gives:

$$
\begin{gathered}
\delta=A \varepsilon^{C \phi} \\
a A \varepsilon{ }^{C \phi}+2 b C A \varepsilon^{C \phi}+C^{2} A \varepsilon^{C \phi}=0 \\
a+2 b C+C^{2}=0
\end{gathered}
$$

which equation has the two roots:

$$
\begin{aligned}
& C_{1}=-b+\sqrt{b^{2}-a} \\
& C_{2}=-b-\sqrt{b^{2}-a}
\end{aligned}
$$

(1) If: $a<0$, or negative, that is, $\beta<a, C_{1}$ is positive and $C_{2}$ negative, and the term with $C_{1}$ is continuously increasing, that is, the synchronous motor is unstable, and, without oscillation, drifts out of step.

(2) If: $0<a<b^{2}$, or $a$ positive, and $b^{2}$ larger than $a$ (that is, the energy consuming term very large), $C^{1}$ and $C^{2}$ are both negative, and, by substituting: $+\sqrt{b^{2}-a}=f$, it is:

$$
C_{1}=-(b-f), \quad C_{2}=-(b+f)
$$

hence:

$$
\delta=A_{1} \varepsilon^{-(b-f) \varphi}+A_{2} \varepsilon^{-(b+f) \phi}
$$

That is, the motor steadies down to its mean position logarithmically, or without any oscillation.

$$
\begin{gathered}
b^{2}<a \\
\text { or: } \quad \frac{\left(c^{2}+p P_{0}\right)^{2}}{16 \pi N M_{0}}<e e_{0} \sin \frac{a-\beta}{z}
\end{gathered}
$$

is the condition under which no oscillation can occur.

As seen, the left side of (34) contains only mechanical, the right side only electrical terms.

$$
a>b^{2} \text {. }
$$

In this case, $\sqrt{b^{2}}-a$ is imaginary, and, substituting:

it is:

$$
g=\sqrt{a-b^{2}}
$$

hence

$$
\begin{aligned}
& C_{1}=-b+j g \\
& C_{2}=-b-j g
\end{aligned}
$$

$$
\delta=\varepsilon^{-b \phi}\left[A_{1} \varepsilon^{+\jmath g \phi}+A_{2} \varepsilon^{-j g \phi}\right]
$$


and, substituting the trigonometric for the exponential functions, gives ultimately:

$$
\delta=B \varepsilon^{-b \phi} \cos (g \varphi+\delta)
$$

That is, the motor steadies down with an oscillation of period:

$$
=\sqrt{\frac{N_{0}=g N}{\frac{N e e_{0} \sin (\alpha-\beta)}{4 \pi z M_{0}}-\frac{\left(c^{2}+p P_{0}\right)^{2}}{\left.64 \pi^{2} M_{0}^{2}\right](36)}}}
$$

and decrement or attenuation constant.

$$
b=\frac{c^{2}+p P_{0}}{8 \pi N M_{0}}
$$

IV.

It follows, however, that under the conditions considered, a cumulative surging, or an oscillation with continuously increasing amplitude, cannot occur, but that a synchronous motor, when displaced in phase from its mean position, returns thereto either aperiodically, if $b^{2}>a$, or with an oscillation of vanishing amplitude, if $b^{2}<a$. At the worst, it may oscillate with constant amplitude, if $b=0$.

Cumulative surging can, therefore, occur only if in the differential equation,

$$
a \delta+2 b \frac{d \hat{o}}{d \varphi}+\frac{d^{2} \delta}{d \varphi^{2}}=0
$$

the coefficient $b$ is negative.

Since $c^{2}$, representing the induction motor torque of the damping device, etc., is positive, and $p P_{0}$ is also positive ( $p$ being the exponent of power variation with speed), this presupposes the existence of a third and negative term $\frac{-h^{2}}{8 \pi N M_{0}}$ in $b$ :

$$
b=\frac{c^{2}+p P_{0}-h^{2}}{8 \pi N M_{0}}
$$

This negative term represents a power:

$$
P_{2}=-h^{2} s
$$

that is, a retarding torque during slow speed, or increasing $\beta$, and accelerating torque during high speed, or decreasing $\beta$.

The source of this torque may be found external to the motor, or internal, in its magnetic circuit. 
External sources of negative $P_{2}$ may be, for instance, the magnetic field of a self-exciting direct current generator, driven by the synchronous motor. With decrease of speed, this field decreases, due to the decrease of generated voltage, and increases with increase of speed. This change of field strength, however, lags behind the exciting voltage and thus speed, that is, during decrease of speed the output is greater than during increase of speed. If this direct current generator is the exciter of the synchronous motor, the effect may be intensified.

The change of power input into the synchronous motor, with change of speed, may cause the governor to act on the prime mover driving the generator, which supplies power to the motor, and the lag of the governor behind the change of output gives a pulsation of the generator frequency, of $e_{0}$, which acts like a negative power $P_{2}$. The pulsation of impressed voltage, caused by the pulsation of $\beta$, may give rise to a negative $P_{2}$ also.

An internal cause of a negative term $P_{2}$ is found in the lag of the synchronous motor field behind the resultant m.m.f. In the preceding discussion, $e$ is the "nominal induced e.m.f." of the synchronous machine, corresponding to the field excitation. The actual magnetic flux of the machine, however, does not correspond to $e$, and thus to the field excitation, but corresponds to the resultant m.m.f. of field excitation and armature reaction, which latter varies in intensity and in phase during the oscillation of $\beta$. Hence, while $e$ is constant, the magnetic flux is not constant, but pulsates with the oscillations of the machine. This pulsation of the magnetic flux lags behind the pulsation of m.m.f., and thereby gives rise to a term in $b$ equation (37). If $P_{0}, \beta, e, e_{0}, Z$ are such that a retardation of the motor increases the magnetizing, or decreases the demagnetizing force of the armature reaction, a negative term $P_{2}$ appears, otherwise a positive term.

$P_{2}$ in this case is the energy consumed by the magnetic cycle of the machine at full frequency, assuming the cycle at full frequency as the same as at frequency of slip $s$.

Or inversely, e may be said to pulsate, due to the pulsation of armature reaction, with the same frequency as $\beta$, but with a phase, which may either be lagging or leading. Lagging of the pulsation of $e$ causes a negative, leading a positive $P_{2}$.

$P_{2}$, therefore, represents the power due to the pulsation of $e$ caused by the pulsation of the armature reaction.

Any appliance increasing the area of the magnetic cycle of 
pulsation, as short-circuits around the field poles, therefore, increases the steadiness of a steady, and increases the unsteadiness of an unsteady synchronous motor.

In self-exciting synchronous converters, the pulsation of $e$ is intensified by the pulsation of direct current voltage caused thereby, and hence of excitation.

Introducing now the term $P_{2}=-h^{2} s$ into the differential equations of Part III., gives the additional cases:

$b<0$, or negative, that is:

$$
\frac{c^{2}+p P_{0}-h^{2}}{8 \pi N M_{0}}<0
$$

Hence, denoting :

gives:

$$
b_{1}=-b=\frac{h^{2}-c^{2}-p P_{0}}{8 \pi N \bar{N} M_{0}}
$$

(4) if:

$$
\begin{array}{lr}
b_{1}^{2}>a: & f=+\sqrt{b_{1}^{2}-a} \\
\delta=A_{1} \varepsilon^{+\left(b_{1}+f\right) \varphi} & +A_{2} \varepsilon^{+b-f) \phi}
\end{array}
$$

That is, without oscillation, the motor drifts out of step, in unstable equilibrium.

(5) if:

$$
\begin{aligned}
& a>b^{2}: \quad g=\sqrt{a-b_{1}{ }^{2}} \\
& \delta=B \varepsilon{ }^{+b_{1} \phi} \cos (g \varphi+\delta)
\end{aligned}
$$

That is, the motor oscillates, with constantly increasing amplitude, until it drops out of step. This is the typical case of cumulative surging by electro-mechanical resonance.

The problem of surging of synchronous machines, and its elimination, thus resolves into the investigation of the coefficient:

$$
b=\frac{c^{2}+p P_{0}-h^{2}}{8 \pi N M}
$$

while the frequency of surging, where such exists, is given by:

$$
N_{0} \sqrt{\frac{\left(N e e_{0} \sin (\alpha-\beta)\right.}{4 \pi z_{0} M_{0}}-\frac{\left(c^{2}+p P_{0}-h^{2}\right)^{2}}{64 \pi^{2} M_{0}^{2}}}
$$

Case (4), steady drifting out of step, has only rarely been observed.

The avoidance of surging thus requires:

(1) An elimination of the term $h^{2}$, or reduction as far as possible. 
(2) A sufficiently large term $c^{2}$ or

(3) A sufficiently large term $p P_{0}$.

(1) refers to the design of the synchronous machine and the system on which it operates. (2) leads to the use of electromagnetic anti-surging devices, as an induction motor winding in the field poles, short-circuits between the poles, or around the poles, and (3) leads to flexible connection to a load or a momentum, as flexible connection with a flywheel, or belt drive of the load.

The conditions of steadiness are:

and if:

$$
\begin{aligned}
& \beta<\alpha \\
& c^{2}+p P_{0}-h^{2}>0
\end{aligned}
$$

$$
\frac{\left(c^{2}+p P_{0}-h^{2}\right)^{2}}{\left(16 \pi N M_{0}\right)}>\frac{e e_{0} \sin (\alpha-\beta)}{z}
$$

no oscillation at all occurs, otherwise an oscillation with decreasing amplitude. 